\title{
YSO accretion shocks: magnetic, chromospheric or stochastic flow effects can suppress fluctuations of X-ray emission
}

\author{
T. Matsakos ${ }^{1,2,3}$, J.-P. Chièze ${ }^{2}$, C. Stehlé ${ }^{3}$, M. González ${ }^{4}$, L. Ibgui ${ }^{3}$, L. de Sá2,3, T. Lanz ${ }^{5}$, S. Orlando ${ }^{6}$, R. Bonito ${ }^{7,6}$, \\ C. Argiroffif ${ }^{7,6}$, F. Reale ${ }^{7,6}$, and G. Peres ${ }^{7,6}$
}

${ }^{1}$ CEA, IRAMIS, Service Photons, Atomes et Molécules, 91191 Gif-sur-Yvette, France e-mail: titos.matsakos@cea.fr

2 Laboratoire AIM, CEA/DSM - CNRS - Université Paris Diderot, IRFU/Service d'Astrophysique, CEA Saclay, Orme des Merisiers, 91191 Gif-sur-Yvette, France

3 LERMA, Observatoire de Paris, Université Pierre et Marie Curie and CNRS, 5 Place J. Janssen, 92195 Meudon, France

${ }^{4}$ Université Paris Diderot, Sorbonne Paris Cité, AIM, UMR 7158, CEA, CNRS, 91191 Gif-sur-Yvette, France

5 Laboratoire Lagrange, Université de Nice-Sophia Antipolis, CNRS, Observatoire de la Côte d'Azur, 06304 Nice Cedex 4, France

${ }^{6}$ INAF - Osservatorio Astronomico di Palermo, Piazza del Parlamento 1, 90134 Palermo, Italy

7 Dipartimento di Fisica e Chimica, Università degli Studi di Palermo, Piazza del Parlamento 1, 90134 Palermo, Italy

Received 2 May 2013 / Accepted 15 July 2013

\begin{abstract}
Context. Theoretical arguments and numerical simulations of radiative shocks produced by the impact of the accreting gas onto young stars predict quasi-periodic oscillations in the emitted radiation. However, observational data do not show evidence of such periodicity. Aims. We investigate whether physically plausible perturbations in the accretion column or in the chromosphere could disrupt the shock structure influencing the observability of the oscillatory behavior.

Methods. We performed local 2D magneto-hydrodynamical simulations of an accretion shock impacting a chromosphere, taking optically thin radiation losses and thermal conduction into account. We investigated the effects of several perturbation types, such as clumps in the accretion stream or chromospheric fluctuations, and also explored a wide range of plasma- $\beta$ values.

Results. In the case of a weak magnetic field, the post-shock region shows chaotic motion and mixing, smoothing out the perturbations and retaining a global periodic signature. On the other hand, a strong magnetic field confines the plasma in flux tubes, which leads to the formation of fibrils that oscillate independently. Realistic values for the amplitude, length, and time scales of the perturbation are capable of bringing the fibril oscillations out of phase, suppressing the periodicity of the emission.

Conclusions. The strength of a locally uniform magnetic field in YSO accretion shocks determines the structure of the post-shock region, namely, whether it will be somewhat homogeneous or if it will split up to form a collection of fibrils. In the second case, the size and shape of the fibrils is found to depend strongly on the plasma- $\beta$ value but not on the perturbation type. Therefore, the actual value of the protostellar magnetic field is expected to play a critical role in the time dependence of the observable emission.
\end{abstract}

Key words. accretion, accretion disks - magnetohydrodynamics (MHD) - radiative transfer - shock waves - instabilities

\section{Introduction}

The accretion process of young stellar objects (YSOs) is believed to take place by funneled streams that originate in the surrounding disk and flow along the field lines of the protostellar magnetosphere (Koenigl 1991; Hartmann et al. 1994; Romanova et al. 2003, 2004, 2008). The gas falls onto the surface of the central body with a supersonic velocity, close to the free-fall speed. Its impact on the chromosphere produces strong shocks of a few million Kelvin, a phenomenon that is observable in X-rays (Ulrich 1976; Gullbring 1994; Calvet \& Gullbring 1998; Lamzin 1998, 1999; Bouvier et al. 2007b). Detailed observations of young stars have detected such emission (Kastner et al. 2002; Schmitt et al. 2005; Günther et al. 2006; Argiroffi et al. 2007; Robrade \& Schmitt 2007; Brickhouse et al. 2010; Dupree et al. 2012), which has to be carefully distinguished from the coronal one. The presence of an accretion shock is probed by a denser $\left(n_{\mathrm{e}} \gtrsim 10^{11} \mathrm{~cm}^{-3}\right)$ and cooler $(T \sim 2-5 \mathrm{MK})$ plasma, as compared to the more tenuous, at least by one order of magnitude, and hotter corona.
The time dependence of the accretion shock's structure is determined by radiative processes, as well as by the topology and strength of the magnetic field (Sacco et al. 2008, 2010; Koldoba et al. 2008; Orlando et al. 2010). The typical field of protostars can be strong enough, on the order of kG (Johns-Krull 2007; Donati et al. 2007), such that it confines the infalling plasma inside magnetic flux tubes and prevents it from escaping sideways. Upon impact, a shock is transmitted into the chromosphere, and a reverse shock propagates along the stream. The simplest local configuration is to assume that the chromosphere is horizontal and threaded by a perpendicular uniform field, as depicted in Fig. 1. Material accumulates vertically and the thickness of the shocked region increases, building up a hot slab of a few million Kelvin. Considering the approximation of an optically thin stream, the hot slab radiates its energy away and collapses within the characteristic cooling time $\left(\sim 10-10^{3} \mathrm{~s}\right)$. This process is expected to repeat quasi-periodically, giving rise to a pulsating emission. Such a cycle has been demonstrated and examined extensively in 1D numerical studies of YSO accretion shocks (Sacco et al. 2008; Koldoba et al. 2008; Sacco et al. 2010). In 


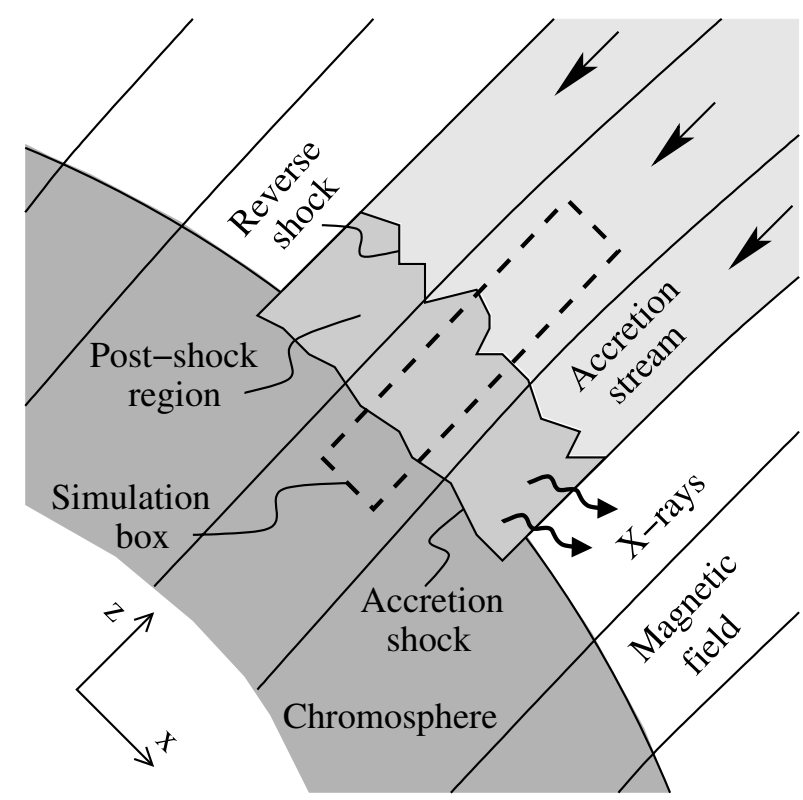

Fig. 1. A schematic low plasma- $\beta$ configuration of an accretion shock. A strong magnetic field penetrates the chromosphere and confines the plasma in flux tubes. The accretion stream impacts on the surface and pushes the chromosphere down to the point where the ram pressure is equal to the stellar pressure. The computational box for the simulations presented in this paper is indicated with a dashed line.

fact, it is a general feature of radiative shocks that the dependence of the cooling function on the temperature may trigger cooling instabilities. In turn, this may lead to the recurrent formation and collapse of the reverse shock, both in the hydrodynamical regime (e.g. Langer et al. 1981; Chevalier \& Imamura 1982; Ramachandran \& Smith 2005b; Sutherland et al. 2003; Mignone 2005) and in the presence of a transverse magnetic field (e.g. Toth \& Draine 1993; Ramachandran \& Smith 2005a).

However, recent observational data of accreting YSOs do not show evidence for such an oscillatory structure (Drake et al. 2009; Günther et al. 2010). In particular, Drake et al. (2009) analyzed the time series of X-ray observations of TW Hya and excluded the presence of periodicity over the frequency range $0.0001-6.811 \mathrm{~Hz}$, which they argue to be the most likely window (the upper limit of the relative amplitude, $A$, is 0.033 for the interval $0.0001-0.192 \mathrm{~Hz}$ and $A=0.056$ for $0.192-6.811 \mathrm{~Hz}$ ). Moreover, Günther et al. (2010) reach a similar conclusion having examined optical and UV data of TW Hya and AA Tau over the frequency ranges $0.02-3 \mathrm{~Hz}(A=0.001)$ and $0.02-50 \mathrm{~Hz}$ $(A=0.005)$, respectively. The absence of periodicity can be attributed to several possible reasons. For instance, by taking radiation transfer effects into account, the cooling of the post-shock region may change and lead to a stable shock stalled inside the chromosphere (Chièze et al., in prep.). On the other hand, complex magnetic field topologies can invalidate the 1D approximation and thus its periodic behavior (Orlando et al. 2013). In a similar context, simulations performed in the high plasma- $\beta$ regime (ratio of the thermal pressure over the magnetic one) indicate a rather smooth emission, thanks to the shocked material that escapes sideways from the accretion column and deflates the hot slab (Orlando et al. 2010). We also note that Sutherland et al. (2003) explored cooling instabilities of radiative shocks in the pure hydro-dynamical 2D case and found that the extra dimension together with small perturbations lead to inhomogeneous shocks. Their filamentary structure enhanced the cooling efficiency and generated turbulence that may introduce ambiguities in the derivation of velocity profiles.

On the other hand, several authors (e.g. Gullbring et al. 1996; Safier 1998; Bouvier et al. 2007a) have pointed out that accretion streams of young stars are clumped and inhomogeneous, while until now only homogeneous accretion flows have been modeled. A detailed multi-dimensional magneto-hydrodynamical (MHD) modeling of a density structured accretion flow impacting on the stellar surface is therefore required to investigate the complex structure and stability of the hot slab for an inhomogeneous stream.

The 1D accretion model that is often found in the literature is based on the assumption of a locally strong and uniform field. By considering typical accretion stream velocities and densities, namely $v_{\text {acc }}=100-500 \mathrm{~km} \mathrm{~s}^{-1}$ and $\rho_{\text {acc }}=10^{-13}-10^{-11} \mathrm{~g} \mathrm{~cm}^{-3}$ (Sacco et al. 2010, and references therein), and a local value of the magnetic field of $B=0.1-2 \mathrm{kG}$ in the impact region, the plasma- $\beta$ value in the post-shock region ${ }^{1}$ is on the order

$\beta_{\mathrm{sh}} \equiv \frac{P_{\mathrm{sh}}}{B^{2} / 8 \pi} \simeq \frac{\rho_{\mathrm{acc}} v_{\mathrm{acc}}^{2}}{B^{2} / 8 \pi} \sim 10^{-2}-10^{2}$,

where we have approximated the thermal pressure of the shocked region, $P_{\mathrm{sh}}$, with the ram pressure of the infalling gas. For the parameter space corresponding to $\beta \lesssim 1$, the plasma is constrained within vertical flux tubes in which case the 1D approximation is justified.

In this paper, we extend the above configuration and we perform 2D MHD simulations. During the evolution, we introduce either density perturbations in the accretion stream, as it is the case for a clumpy flow, or pressure variabilities in the chromosphere, similar to what is observed in the sun. We study the time dependence of the shock structure for several plasma- $\beta$ values, looking for multi-dimensional mechanisms that could disrupt or even suppress the oscillation. We note that the presence of a strong magnetic field constrains significantly the plasma dynamics; for instance it can prevent the generation of large scale chaotic motion such as that observed in Sutherland et al. (2003).

The paper is structured as follows. Section 2 decribes the numerical setup and lists the simulation models. Section 3 derives simple analytical expressions to estimate the effects that perturbations can have on the temporal shock evolution. Section 4 presents our numerical results and discusses their relevance to real accretion shocks. Finally, Sect. 5 summarizes our work and reports our conclusions.

\section{Theoretical framework}

\subsection{The $M H D$ equations}

The MHD perfect gas equations, including the appropriate terms suited for the study of accretion shocks, are

$$
\begin{aligned}
& \frac{\partial \rho}{\partial t}+\nabla \cdot(\rho \boldsymbol{v})=0, \\
& \frac{\partial \boldsymbol{v}}{\partial t}+(\boldsymbol{v} \cdot \nabla) \boldsymbol{v}+\frac{1}{4 \pi \rho} \boldsymbol{B} \times(\nabla \times \boldsymbol{B})+\frac{1}{\rho} \nabla P=\boldsymbol{g}, \\
& \frac{\partial P}{\partial t}+\boldsymbol{v} \cdot \nabla P+\gamma P \nabla \cdot \boldsymbol{v}=-(\gamma-1)\left(\nabla \cdot \boldsymbol{F}+n_{\mathrm{e}} n_{\mathrm{H}} \Lambda\right), \\
& \frac{\partial \boldsymbol{B}}{\partial t}+\nabla \times(\boldsymbol{B} \times \boldsymbol{v})=0 .
\end{aligned}
$$

1 In the following, the plasma- $\beta$ value will always refer to the postshock region. 
T. Matsakos et al.: Suppressing the periodic emission of YSO accretion shocks

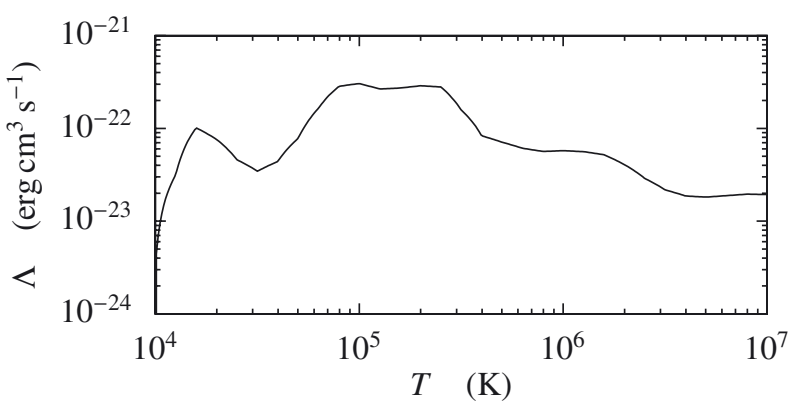

Fig. 2. Adopted cooling function, $\Lambda$, as a function of the temperature. The negative slope in the temperature range $T=10^{5}-10^{7} \mathrm{~K}$ is triggering cooling instabilities: the more the plasma cools the higher the energy losses are.

The equations are written in cgs units for the variables, $\rho, P$, and $\boldsymbol{v}$, which denote the gas density, pressure, and velocity, respectively. The magnetic field, $\boldsymbol{B}$, also satisfies the condition $\nabla \cdot \boldsymbol{B}=0$. The ratio of specific heats is $\gamma=5 / 3$ and $\boldsymbol{g}$ represents gravity. The thermal conduction flux is given by

$\boldsymbol{F}=\frac{F_{\text {sat }}}{F_{\text {sat }}+\left|\boldsymbol{F}_{\mathrm{Spi}}\right|} \boldsymbol{F}_{\mathrm{Spi}}$

where $F_{\text {sat }}=5 \rho c_{\mathrm{s}}^{3}$ is the saturated flux (Cowie \& McKee 1977) and $c_{\mathrm{S}}$ the sound speed. The term $\boldsymbol{F}_{\text {Spi }}$ consists of two components, one along $(\|)$ and one across $(\perp)$ the magnetic field lines (Spitzer 1962), given by

$\boldsymbol{F}_{\mathrm{Spi}}=\kappa_{\|} \frac{(\boldsymbol{B} \cdot \nabla T) \boldsymbol{B}}{\boldsymbol{B}^{2}}+\kappa_{\perp}\left(\nabla T-\frac{(\boldsymbol{B} \cdot \nabla T) \boldsymbol{B}}{\boldsymbol{B}^{2}}\right)$,

where $T$ is the temperature, $n_{\mathrm{H}}$ is the number density of hydrogen atoms, and $k_{\mathrm{B}}$ is the Boltzmann constant. The thermal conduction coefficients are given by $\kappa_{\|}=-9.2 \times 10^{-7} T^{5 / 3}$ and $\kappa_{\perp}=-3.3 \times 10^{-16} n_{\mathrm{H}}^{2} /\left(T^{1 / 2} \boldsymbol{B}^{2}\right)$, respectively, both of which are in units of $\mathrm{erg} \mathrm{s}^{-1} \mathrm{~K}^{-1} \mathrm{~cm}^{-1}$. A mean atomic weight of $\mu=1.2$ is considered, i.e. $\rho=\mu n_{\mathrm{H}} m_{\mathrm{H}}$, where $m_{\mathrm{H}}$ is the hydrogen atomic mass. The electron number density is denoted with $n_{\mathrm{e}}$ and the gas is assumed to be always fully ionized. The latter is a valid approximation for the post-shock region, the cooling of which plays a key role in this study. The pre-shocked material is cold enough such that the energy losses are negligible and the actual value of $n_{\mathrm{e}}$ is not important there.

The cooling function, $\Lambda$, is adopted from Orlando et al. (2010) and is shown in Fig. 2. It depends on the temperature and represents radiative losses of an optically thin plasma. The profile was derived with the PINTofALE spectral code (Kashyap \& Drake 2000) using the APED V1.3 atomic line database (Smith et al. 2001) and considering metal abundances 0.5 times the solar ones (Telleschi et al. 2007). This abundance has been choosen because some observations tend to indicate that the accretion flow is depleted in metals (Stelzer \& Schmitt 2004; Drake et al. 2005). Finally, in order to prevent the chromosphere from cooling, we define a temperature threshold, $T_{\text {cut }}=10^{4} \mathrm{~K}$, below which $\Lambda$ is set to zero.

Finally, we make use of a flow tracer, $Q$, which is a passive scalar that obeys the advection equation: $\partial Q / \partial t+v \cdot \nabla Q=0$. It does not interfere with the dynamics but simply follows the motion of the plasma and helps visualize its path.

\subsection{Numerical setup}

The simulations are carried out with $\mathrm{PLUTO}^{2}$, a numerical code for computational astrophysics (Mignone et al. 2007, 2012). We adopt the ROE solver for the integration and we apply second order accuracy on both space and time.

The configuration is simulated in 2D cartesian coordinates ${ }^{3}$, $(x, z)$, neglecting surface curvature and stellar rotation, as shown in Fig. 1. We focus on the interior of the stream in order to remain as close as possible to the 1D problem. Therefore, we avoid influence from the effects at the interface between the corona and the funnel flow (e.g. Orlando et al. 2010). A chromosphere is initialized on the lower part of the computational box, underneath a hot corona (e.g. Sacco et al. 2008, 2010; Orlando et al. 2010). Gravity is assumed uniform, $\boldsymbol{g}=-\left(G M / R^{2}\right) \hat{z}$, with the mass and radius of the star equal to those of MP Mus, i.e. $M=1.2 M_{\odot}$ and $R=1.3 R_{\odot}$ (Argiroffi et al. 2007). For simplicity, both the chromosphere and the corona are assumed isothermal, with temperatures, $T_{\text {chr }}=10^{4} \mathrm{~K}$ and $T_{\text {cor }}=10^{6} \mathrm{~K}$, respectively. The pressure and density profiles of each one have an exponential dependence such that the configuration is in equilibrium and pressure is continuous. We note that during the first steps of the simulations the corona is readily engulfed by the accretion stream and hence its presence is not important for this study. Finally, a uniform vertical magnetic field, $\boldsymbol{B}=B_{0} \hat{z}$, is initialized throughout the box and is evolved self-consistently during the simulation.

The accretion stream is not initially present in the domain but enters from the top. In particular, we prescribe the properties of the infalling plasma on the upper boundary, namely, velocity $v_{\text {acc }}=500 \mathrm{~km} \mathrm{~s}^{-1}$, number density $n_{\mathrm{acc}}=10^{11} \mathrm{~cm}^{-3}$ and temperature $T_{\text {acc }}=10^{3} \mathrm{~K}$ (e.g. Bary et al. 2008). These values, along with $B$, are kept fixed during the simulation, but see also Sect. 2.3 for more details on the applied perturbation. At the ghost cells of the bottom boundary, we specify zero velocities, we set the magnetic field equal to its initial uniform value, and we apply the isothermal equilibrium profiles for the density and pressure. Moreover, by fixing the physical quantities to a given value implies that MHD waves might be reflected. We have investigated the effect of such boundary conditions and found that they can be neglected. This can be seen from the reference simulation NoPert which reproduces the 1D oscillatory behavior reported in the literature (see Sect. 3.1). Lastly, at the left and right edges of the box we apply periodic boundary conditions.

The computational domain spans $x=\left[0.0,0.5 \times 10^{-3}\right] R_{\odot}$ in the horizontal direction and $z=\left[0.0,7.7 \times 10^{-2}\right] R_{\odot}$ in the vertical, and is resolved by a static grid of $[128 \times 2048]$ cells. We have not made use of Adaptive Mesh Refinement (AMR) since the nature of the problem is such that most parts of the computational domain require a high level of refinement. Besides, we also avoid the spurious perturbations that could be introduced by a dynamically evolving grid and would interfere with those applied physically in the system. Note that due to the significant elongation of the simulation box, all spatial distribution plots expand the horizontal direction by a factor of 2 and hence the figures look vertically compressed. The final time of the simulations is on the order of 5 or $10 \mathrm{ks}$, depending on the case under consideration, and corresponds approximately to 5-10 shock oscillations of the $1 \mathrm{D}$ case. Each simulation requires from a few to several thousands hours of computational time to complete, due to constraints imposed on the time step by the optically thin radiation

\footnotetext{
2 Freely available at http://plutocode.ph.unito.it

3 The unit vectors are denoted with $\hat{x}$ and $\hat{z}$.
} 

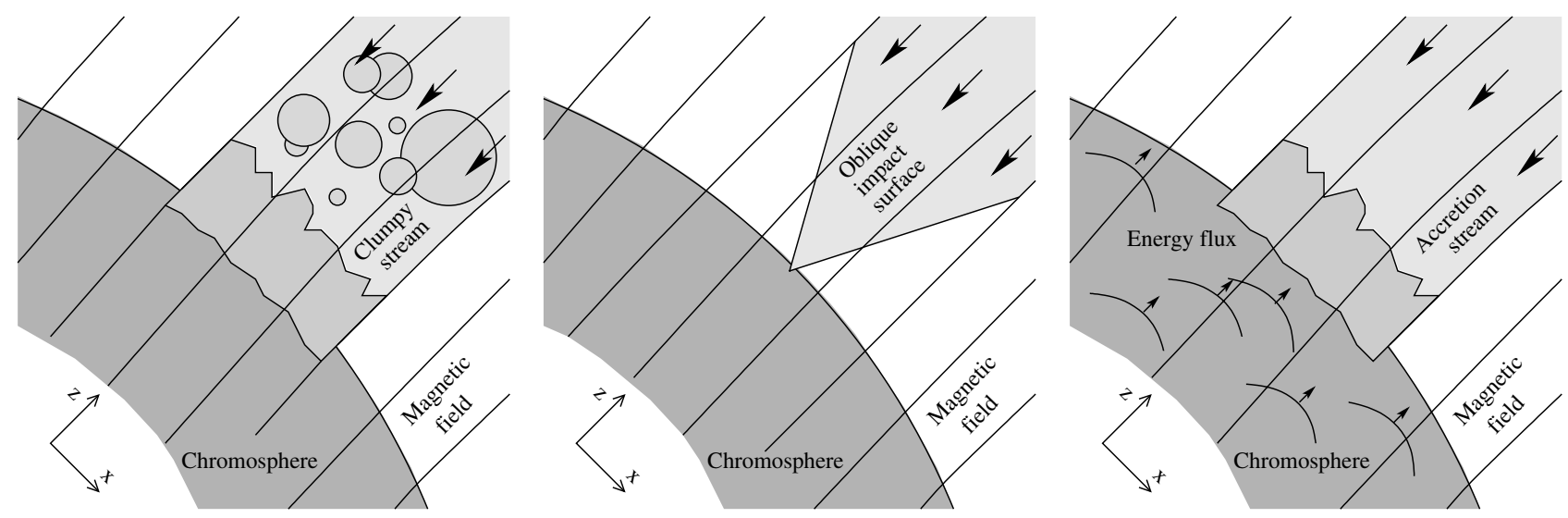

Fig. 3. Types of perturbation considered: a clumpy structure of the accretion stream (left; models Rnd\#, IsSm\#/IsLg\#, ClSm\#/ClLg\#), an oblique impacting surface (middle; model $0 \mathrm{bl500}$ ), and an energy flux present in the chromosphere (right; models ChrFlx\#).

Table 1. Numerical models.

\begin{tabular}{lccccccc}
\hline \hline Name & $B_{0}(\mathrm{G})$ & Amplitude & Length scale & Time scale & Location & Perturbation description & Final time \\
\hline Rnd20 & 20 & 0.01 & $25 \mathrm{~km}$ & $0.05 \mathrm{~s}$ & Accretion stream & Random density perturbations & $7 \mathrm{ks}$ \\
Rnd50 & 50 & 0.01 & $25 \mathrm{~km}$ & $0.05 \mathrm{~s}$ & Accretion stream & Random density perturbations & $7 \mathrm{ks}$ \\
Rnd100 & 100 & 0.01 & $25 \mathrm{~km}$ & $0.05 \mathrm{~s}$ & Accretion stream & Random density perturbations & $7 \mathrm{ks}$ \\
Rnd250 & 250 & 0.01 & $25 \mathrm{~km}$ & $0.05 \mathrm{~s}$ & Accretion stream & Random density perturbations & $7 \mathrm{ks}$ \\
Rnd500 & 500 & 0.01 & $25 \mathrm{~km}$ & $0.05 \mathrm{~s}$ & Accretion stream & Random density perturbations & $7 \mathrm{ks}$ \\
\hline IsSm20 & 20 & 10 & $300 \mathrm{~km}$ & $30 \mathrm{~s}$ & Accretion stream & Isolated small clumps & $4 \mathrm{ks}$ \\
IsSm500 & 500 & 10 & $300 \mathrm{~km}$ & $30 \mathrm{~s}$ & Accretion stream & Isolated small clumps & $4 \mathrm{ks}$ \\
IsLg20 & 20 & 10 & $1000 \mathrm{~km}$ & $2 \mathrm{~min}$ & Accretion stream & Isolated large clumps & $4 \mathrm{ks}$ \\
IsLg500 & 500 & 10 & $1000 \mathrm{~km}$ & $2 \mathrm{~min}$ & Accretion stream & Isolated large clumps & $4 \mathrm{ks}$ \\
\hline ClSm20 & 20 & 10 & $300 \mathrm{~km}$ & $0.5 \mathrm{~s}$ & Accretion stream & Stream of small clumps & $4 \mathrm{ks}$ \\
ClSm500 & 500 & 10 & $300 \mathrm{~km}$ & $0.5 \mathrm{~s}$ & Accretion stream & Stream of small clumps & $4 \mathrm{ks}$ \\
ClLg20 & 20 & 10 & $1000 \mathrm{~km}$ & $1.5 \mathrm{~s}$ & Accretion stream & Stream of large clumps & $4 \mathrm{ks}$ \\
ClLg500 & 500 & 10 & $1000 \mathrm{~km}$ & $1.5 \mathrm{~s}$ & Accretion stream & Stream of large clumps & $4 \mathrm{ks}$ \\
\hline Obl500 & 500 & - & $3500 \mathrm{~km}$ & $15 \mathrm{~min}$ & Accretion stream & Oblique impacting surface & $7 \mathrm{ks}$ \\
\hline ChrFlx20 & 20 & $10 F_{\odot}$ & $3500 \mathrm{~km}$ & $15 \mathrm{~min}$ & Chromosphere & Energy flux & $5 \mathrm{ks}$ \\
ChrFlx100 & 100 & $10 F_{\odot}$ & $3500 \mathrm{~km}$ & $15 \mathrm{~min}$ & Chromosphere & Energy flux & $5 \mathrm{ks}$ \\
ChrFlx500 & 500 & $10 F_{\odot}$ & $3500 \mathrm{~km}$ & $15 \mathrm{~min}$ & Chromosphere & Energy flux & $5 \mathrm{ks}$ \\
\hline NoPert & 20 & - & - & - & & - & No perturbation \\
\hline
\end{tabular}

Notes. Their code names summarize the type of perturbation and the strength of the magnetic field.

cooling and thermal conduction. This prevented us from exploring the simulations for longer times.

\subsection{Models and the applied perturbations}

Table 1 lists the simulations that have been carried out, each one assuming a different type of perturbation which is visualized in Fig. 3. In general, we investigate five broad classes of models, a) one that introduces random density perturbations in the accretion stream; b) one that assumes isolated clumps in the infalling material; c) one that considers a fully clumped stream; d) one having an oblique surface of first impact; and e) one that includes variability in the chromosphere. For the density fluctuations of the infalling gas, we explore the minimum and maximum spatial scales that are defined by the resolution and size of the computational box, respectively.

In particular, the models Rnd\# consider random density perturbations of $\sim 1 \%$ that take place at the cell level, or equivalently, at a scale of $\sim 25 \mathrm{~km}$. This is done by prescribing the density at the upper boundary in the following way:

$\rho=\rho_{\mathrm{acc}}+\delta \rho_{\mathrm{acc}}=\left(1+q_{\mathrm{rand}}\right) \rho_{\mathrm{acc}}$, where $q_{\text {rand }}$ is a random number that follows a uniform distribution in the range $[-0.01,0.01]$. The value of $q_{\text {rand }}$ is different for each ghost cell and it is randomized repeatedly with a period of $\Delta t_{\text {rand }}=\Delta z / v_{\text {acc }} \simeq 0.05 \mathrm{~s}$. This interval corresponds to the time required for the stream to travel a distance equivalent to the cell height, $\Delta z$.

The models IsSm\# and IsLg\# consider isolated clumps in the accretion stream, which are incorporated by adopting a Gaussian profile with a constant effective width of $\sigma_{0}$, a randomized location $x_{0}$, and a randomized time of appearance $t_{0}$. Specifically, the density at the upper boundary condition is set as

$\rho=\rho_{\mathrm{acc}}+\delta \rho_{\mathrm{acc}}$

$$
=\left[1+q_{\mathrm{cl}} \exp \left(-\frac{\left(x-x_{0}\right)^{2}+v_{\mathrm{acc}}^{2}\left(t-t_{0}\right)^{2}}{\sigma_{0}^{2}}\right)\right] \rho_{\mathrm{acc}},
$$

where $q_{\mathrm{cl}}=10$ is the peak density contrast and $t_{0}$ is the time that the clump enters the computational box. The value of $t_{0}$ is chosen in such a way that the clump is initialized far away from the boundary. New values are given to $x_{0}$ and $t_{0}$ every $\Delta t_{\mathrm{cl}}$, a time interval large enough to ensure that the boundary density is equal to $\rho_{\text {acc }}$ before each new randomization. Note that a small randomization applies also to $\Delta t_{\mathrm{cl}}$ in order to avoid an exactly 
periodic appearance of clumps. For the case of large clumps, IsLg\#, the parameters are $\sigma_{0}=1000 \mathrm{~km}$ and $\Delta t_{\mathrm{cl}}=2 \mathrm{~min}$, whereas for the case of small clumps, IsSm\#, the parameters are $\sigma_{0}=300 \mathrm{~km}$ and $\Delta t_{\mathrm{cl}}=30 \mathrm{~s}$.

The models ClSm\# and ClLg\# describe a fully clumped stream using the same gaussian distribution for the overdensities as for the isolated clumps, with the difference that the clumps appear so frequently that they may overlap. In particular, new values are given to $x_{0}$ and $t_{0}$ every $\Delta t_{\mathrm{cl}}=\sigma_{0} / v_{\mathrm{acc}}$, which is equivalent to a vertical clump spacing comparable to their radius. Moreover, in order to simulate an accretion stream that is overall described by the value $\rho_{\text {acc }}$, we set the peak density of the gaussian distribution equal to that value. For numerical reasons, we apply a background uniform density which is 10 times lower than $\rho_{\text {acc }}$.

For the case of an oblique impacting surface, the assumption of periodic boundaries in the horizontal direction is only valid in the low plasma- $\beta$ regime, where the magnetic field can effectively confine the plasma and prevent it from escaping sideways. Therefore, we explore just one model for a large value of $\boldsymbol{B}$, Ob1500. To achieve an oblique impacting surface, we define the accreting gas variables only at the part of the top boundary that satisfies the condition:

$\left|x-\frac{x_{\max }}{2}\right|<\frac{t}{t_{\text {fill }}} \frac{x_{\max }}{2}$,

where $x_{\max }$ is the width of the computational box and $t_{\text {fill }}=$ $15 \mathrm{~min}$ is the time that the stream needs to fill up the whole domain. At each time step, the cross section of the stream increases giving a conical shape to the impacting surface, with the value of $t_{\text {fill }}$ effectively controlling its aperture.

On the other hand, models ChrFlx\# consider a homogeneous accretion stream, and instead perturb the system from the lower edge of the computational box, i.e. the chromosphere. In particular, we introduce an energy flux, of a similar type to what is observed in the sun, by specifying a pressure variation on the bottom boundary that depends on both time and space:

$P=P_{\mathrm{chr}}+\delta P_{\mathrm{chr}}=P_{\mathrm{chr}}+\frac{F_{\mathrm{chr}}}{c_{\mathrm{chr}}} \sin \left(\frac{2 \pi t}{t_{\mathrm{chr}}}-\frac{2 \pi x}{L}\right)$.

In the above expression, $c_{\mathrm{chr}}$ is the chromospheric sound speed, $F_{\text {chr }}=5 \times 10^{9} \mathrm{erg} \mathrm{cm}^{-2} \mathrm{~s}^{-1}$ is the energy flux assumed, $t_{\mathrm{chr}}=$ $15 \mathrm{~min}$ is the periodicity of the variation, and $L=3500 \mathrm{~km}$ its length scale (e.g. Bohn 1984; Judge 2006; Bello González et al. 2009; Beck et al. 2009, 2013). We have chosen a value for $F_{\mathrm{chr}}$ that is an order of magnitude larger than the solar one because young stars are in general expected to be more active than the sun. In addition, note that the assumed periodicity time scale of the energy flux is a few times larger than that of the shock oscillation time, which suggests that a value closer to the solar one, i.e. a few minutes, would perturb more strongly the system. We also apply an appropriate variability to the density at the bottom boundary such that the chromosphere remains isothermal and hence we avoid thermal conduction effects that could diffuse the perturbation. We note that a purely temporal fluctuation, equivalent to a 1D problem, is also relevant to the study of its effect on the shock oscillations. However, such cases are explored in de Sá et al. (in prep.) and hence we do not discuss them here.

The simulations are performed in both a high and a low plasma- $\beta$ regime, and in particular for magnetic fields ranging between $20 \mathrm{G}$ and $500 \mathrm{G}$, or equivalently for post-shock $\beta$ values from $\beta \lesssim 100$ to $\beta \gtrsim 0.01$. This enables to isolate the hydrodynamical effects and expose the richness of the MHD formulation.

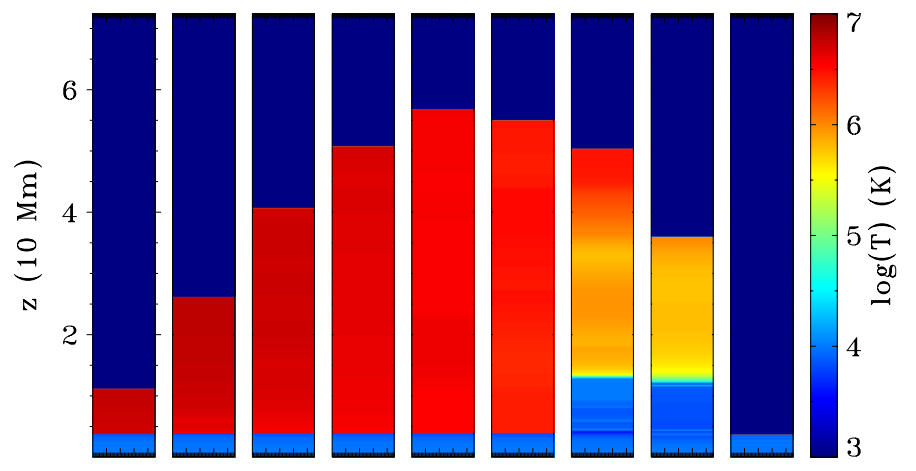

Fig. 4. Snapshots of the logarithmic temperature distribution during one oscillation of the reverse shock for the model NoPert. The dark blue color corresponds to the accretion stream, the light blue to the chromosphere and the red to the hot post-shock region which gradually cools down. The panels are separated by $108 \mathrm{~s}$, showing the formation and recollapse of the post-shock region due to the cooling instabilities induced by the optically thin radiation cooling.

Finally, the model NoPert is a $2 \mathrm{D}$ representation of the $1 \mathrm{D}$ problem assuming a homogeneous accretion stream and a static chromosphere. It is the reference case in order to present the basic features of the shock evolution. We have chosen the value of $20 \mathrm{G}$.

\section{Analytical approach}

\subsection{Description of the equivalent $1 D$ problem}

The typical evolution of 1D accretion shocks, in the context of YSOs (Sacco et al. 2008, 2010; Koldoba et al. 2008), can be summarized by discussing model NoPert. Figure 4 shows the formation and collapse of the post-shock region. The impact of the accretion stream compresses the gas, heating it to temperatures of a few MK. The post-shock region steadily increases in height as the reverse shock travels upwards along the funnel. However, the optically thin radiation losses of the hot slab are significant, effectively cooling down the plasma. Within the characteristic cooling time, the temperature drops by almost 3 orders of magnitude and thus the pressure can no longer support the infalling gas. In turn, the post-shock region collapses, repositing material onto the chromosphere. Subsequently, a new hot slab starts to build up and the process is repeated. Since the $\mathrm{X}$-ray emission depends on the density, temperature and volume of the hot plasma, the emitted radiation of the accretion shock will show a quasi-periodic temporal structure.

In fact, this can be seen in Fig. 5, which displays the quantity:

$\langle T(t, z)\rangle=\frac{\int \rho^{2} T \mathrm{~d} x}{\int \rho^{2} \mathrm{~d} x}$.

This emission-measure weighted temperature is calculated at each $t$ and $z$ and provides an estimate of the time dependence of the expected radiation (Orlando et al. 2010). Even though the integration in the $x$ direction is not important for this $x$-symmetric simulation, it is particularly useful in the general 2D cases. Evidently, the corresponding observable flux of model NoPert is intermittent with an average periodicity of approximately a thousand seconds. The small variation to the maximum height that the reverse shock reaches at each oscillation, i.e. between 5 and $7 \mathrm{Mm}$, is attributed to the MHD waves that propagate in the post-shock region and are reflected at the bottom boundary and the shock surfaces. These perturbations, that are independent 


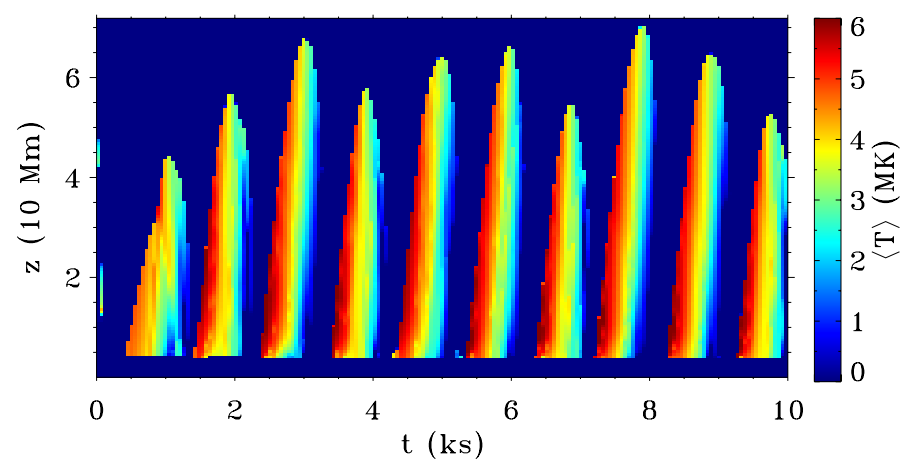

Fig. 5. The vertical structure of the emission-measure weighted temperature as a function of time. Cooling instabilities induce a quasi-periodic structure in the expected radiation with a period of roughly a thousand seconds.

of $x$, create density peaks in the hot slab which in turn affect the time at which the cooling instabilities are triggered. For instance, the rightmost panels of Fig. 4 show that the vertical profile of the temperature during the collapse phase is variable. Moreover, the cooling function adopted in our simulations is not smooth, as it can be seen from Fig. 2, and hence its features in the presence of waves may also contribute to producing nonidentical collapse phases. Nevertheless, these are second order effects in the simulations and are neglected for the rest of this study.

\subsection{Discussion on the qualitative effects of perturbations}

In this context, the question that arises is to what extent perturbations can disrupt this oscillatory stucture, and especially whether its periodic behavior is suppressed.

The key quantity that determines the evolution of the system is the characteristic cooling time, $\tau_{\text {cool }}$. The $1 \mathrm{D}$ approximation assumes that a unique value of $\tau_{\text {cool }}$ describes the whole postshock region, a fact that results in the quasi-periodic global behavior described above. However, if adjacent vertical flux tubes have either a small phase difference in their oscillations or a slightly different $\tau_{\text {cool }}$ value, then a significant horizontal gradient in the pressure of the post-shock region would develop due to the asychronous collapse.

In the case of a weak magnetic field, or equivalently in the high plasma- $\beta$ regime, this will create large velocity components along the $x$ direction that subsequently will lead to a chaotic plasma motion. The density inhomogeneities of the post-shock region will enhance the energy losses, shortening also the cooling time (Sutherland et al. 2003). On the other hand, the presence of a relatively strong vertical magnetic field will prevent horizontal interaction. For instance, an out-of-phase collapse of neighboring flux tubes will still retain their independent evolution ${ }^{4}$. Following Sacco et al. (2010) and Orlando et al. (2010), who have briefly described this mechanism, we refer to these magnetically confined vertical structures as fibrils. Hence, in the low plasma- $\beta$ case, these fibrils can be approximated as isolated quasi-periodic emitters and the overall X-ray radiation is simply the sum of their individual contribution. Therefore, an out-ofphase oscillation would smooth out any global periodic signature in the observed flux, even though each fibril would follow the 1D quasi-periodic evolution described in Sect. 3.1.

\footnotetext{
4 In the latter argument, we have neglected communication by MHD waves because they do not affect $\tau_{\text {cool }}$ considerably.
}

\subsection{Quantitative study of the fibril structure}

In order to quantify the problem we first derive some simplified expressions for the fibril structure.

\subsubsection{The cooling time scale of fibrils}

A lower limit of the Mach number of the accreting gas is found by assuming a relatively small infalling speed, $v_{\mathrm{acc}}=100 \mathrm{~km} \mathrm{~s}^{-1}$, for a gas of a temperature, $T_{\mathrm{acc}}=1000 \mathrm{~K}$ :

$M_{\mathrm{acc}}=\frac{v_{\mathrm{acc}}}{c_{\mathrm{acc}}} \simeq \frac{v_{\mathrm{acc}}}{\sqrt{5 k_{\mathrm{B}} T_{\mathrm{acc}} / 3 \mu m_{\mathrm{H}}}}>15$,

where $c_{\mathrm{acc}}$ is the sound speed in the accretion stream and $k_{\mathrm{B}}$ the Boltzmann constant. Given this high value, the shock can be generally assumed strong and hence its postshock temperature is proportional to the velocity squared:

$T_{\mathrm{sh}} \propto v_{\mathrm{acc}}^{2}$.

Moreover, the optically thin cooling time, $\tau_{\text {cool }}$, can be estimated from Eq. (4), since the plasma pressure changes by $\sim P_{\mathrm{sh}}$ in that interval:

$$
\frac{P_{\mathrm{sh}}}{\tau_{\mathrm{cool}}} \propto \rho_{\mathrm{acc}}^{2} \Lambda \propto \rho_{\mathrm{acc}}^{2} T_{\mathrm{sh}}^{-1 / 2}
$$

Here we have used the expression $\Lambda \propto T^{-1 / 2}$ as a rough approximation in the temperature range $10^{5}-10^{7} \mathrm{~K}$, see Fig. 2 . Combining the above relations and given the fact that the kinetic energy of the accretion stream is converted to thermal pressure upon impact, $P_{\mathrm{sh}} \simeq \rho_{\mathrm{acc}} v_{\mathrm{acc}}^{2}$, the cooling time is found to have the following dependence (e.g. Sacco et al. 2010):

$\tau_{\mathrm{cool}} \propto \frac{v_{\mathrm{acc}}^{3}}{\rho_{\mathrm{acc}}}$.

\subsubsection{The length scales of the fibrils}

Let $h$ be the maximum height that the post-shock region attains and $d$ the width of the fibrils. The quantity $h$ can be estimated by multiplying the time that the reverse shock propagates, $\tau_{\text {cool }}$, with its velocity, which is proportional to $v_{\text {acc }}$ (e.g. Sacco et al. 2010):

$h \propto v_{\mathrm{acc}} \tau_{\mathrm{cool}} \propto \frac{v_{\mathrm{acc}}^{4}}{\rho_{\mathrm{acc}}}$.

The width of the fibrils is relevant only in the low plasma- $\beta$ regime, because otherwise the magnetic flux tubes cannot constrain the plasma dynamics, and the infalling gas is effectively mixed after the impact. In the former case, we can estimate $d$ from the horizontal component of Eq. (3) by assuming a small deformation of the flux tube and decomposing the magnetic field as $\boldsymbol{B}=b_{x} \hat{\boldsymbol{x}}+\left(B_{0}+b_{z}\right) \hat{z}$, with $b_{x}, b_{z} \ll B_{0}$ :

$\frac{\delta P}{\delta x} \simeq \frac{B_{0}}{4 \pi}\left(\frac{\delta b_{x}}{\delta z}-\frac{\delta b_{z}}{\delta x}\right)$

The first term of the right hand side represents the magnetic tension due to the curvature of the magnetic fieldlines, $\delta b_{x} / \delta z \sim$ $b_{x} /(h / 2)$, and the second term represents the gradient of the magnetic pressure. We neglect the latter, since we do not expect a net difference in $b_{z}$ between the inside and outside of the fibril. Therefore,

$\frac{P_{\mathrm{sh}}}{d} \sim \frac{B_{0}}{4 \pi} \frac{b_{x}}{h / 2} \sim \frac{B_{0}^{2}}{8 \pi} \frac{4 d}{h^{2}}$, 
where in the last relation we used the property of the tangent, i.e. $b_{x} / B_{0} \simeq(d / 2) /(h / 2)$. Finally, we obtain:

$d \propto \sqrt{\beta} h$.

This expression relates the width of the fibril with its maximum height as well as the plasma- $\beta$.

\subsubsection{Hydrodynamical limit, no fibrils}

In the high plasma- $\beta$ regime, the time scale for the homogenization of the post-shock pressure is on the order of $r_{\mathrm{acc}} / c_{\mathrm{sh}} \simeq 500 \mathrm{~s}$. Here we have assumed a fraction of the solar radius for the accretion stream cross section, $r_{\text {acc }} \sim 10^{5} \mathrm{~km}$, and a post-shock sound speed of $c_{\mathrm{sh}} \sim 200 \mathrm{~km} \mathrm{~s}^{-1}$. This time scale is comparable to $\tau_{\text {cool }}$ which suggests that any potential phase differences between adjacent vertical elements of the hot slab are likely to smooth out. As a result, there are no fibrils forming and the accretion shock is expected to maintain its periodic behavior in the high plasma- $\beta$ regime.

\subsection{The effects of perturbations}

In the context of the simplified configuration of Fig. 1, the disruption of the global shock periodicity comes down to the search of a potential mechanism that can introduce a net and adequate phase difference in the oscillations of adjacent flux tubes, or equivalently a gradient in their $\tau_{\text {cool }}$ value. In general, perturbations are characterized by three quantities, a) amplitude; b) length scale and c) time scale. Since any small phase difference between fibrils will not increase further in the absence of perturbations, their amplitude cannot be arbitrarily small. However, a continuous perturbation may have cumulative results, and thus even small variabilities might be adequate, as long as they do not cancel each other's effects in time. Moreover, their spatial extent should be larger than the fibril width, $d$, otherwise the contained post-shock region can be homogenized. Finally, the temporal scale of the perturbation should be comparable to the cooling time, $\tau_{\text {cool }}$.

Below, we focus on the case of a strong magnetic field and we estimate the effects of general types of perturbations (Fig. 3) to the temporal evolution of the shock structure.

\subsubsection{Density fluctuations in the accretion stream}

A radial density profile across the accretion column will inevitably destroy the global periodicity due to the different resulting cooling times of each vertical element (Bonito et al., in prep.). However, here we do not account for such initial conditions, but instead we study the perturbed uniform profile. By considering small variations to the infalling gas density, since the velocity is not expected to fluctuate considerably, Eq. (16) gives:

$\tau_{\mathrm{cool}} \propto \frac{1}{\rho_{\mathrm{acc}}+\delta \rho_{\mathrm{acc}}} \simeq \frac{1}{\rho_{\mathrm{acc}}}\left[1-\frac{\delta \rho_{\mathrm{acc}}}{\rho_{\mathrm{acc}}}+\left(\frac{\delta \rho_{\mathrm{acc}}}{\rho_{\mathrm{acc}}}\right)^{2}\right]$.

If the density perturbations are random, one has: $\rho_{\mathrm{acc}} \pm \delta \rho_{\mathrm{acc}}$. To first order, their effects cancel each other and $\tau_{\text {cool }}$ remains unchanged. However, by taking second order terms into account, a net change does appear in the cooling time. Nevertheless, the average value of $\tau_{\text {cool }}$ is affected globally, i.e. at all $x$, and hence the system will always be in phase. Consequently, we should rather focus on lasting phase shifts that can be introduced between adjacent fibrils, namely a local variation of $\delta \rho_{\text {acc }}$. Rewriting Eq. (16) we obtain:

$\frac{\delta \tau_{\mathrm{cool}}}{\tau_{\mathrm{cool}}} \propto-\frac{\delta \rho_{\mathrm{acc}}}{\rho_{\mathrm{acc}}}$

Therefore, a clump with a significant density constrast can considerably affect the local cooling time, within a range comparable to its size. Note that the presence of several clumps, apart from bringing the fibrils out of phase faster, could also reduce the required amplitude of the perturbations due to their cumulative effect.

\subsubsection{Perturbations in the chromosphere}

A variation of $\delta P_{\mathrm{chr}}$ and $\delta \rho_{\mathrm{chr}}$ may relocate locally and temporarily the depth down to which the accretion shock can penetrate into the chromosphere, as well as influence the postshock cooling efficiency. Since pressure fluctuations $\left(\delta P_{\mathrm{chr}} \sim\right.$ $5 \times 10^{3} \mathrm{erg} \mathrm{cm}^{-3}$ ) are larger than the ram pressure of the accretion shock $\left(\rho_{\mathrm{acc}} v_{\mathrm{acc}}^{2} \sim 300 \mathrm{erg} \mathrm{cm}^{-3}\right)$, the activity of the chromosphere is capable of influencing its evolution. In fact, notice that even if we consider the solar energy flux, which is one order of magnitude lower, the value of $\delta P_{\text {chr }}$ would still be on the same order as $\rho_{\mathrm{acc}} v_{\mathrm{acc}}^{2}$. Moreover, this kind of variability is on the order of minutes with the typical lengths involved being a few thousand km (e.g. Bohn 1984; Judge 2006; Bello González et al. 2009; Beck et al. 2009, 2013). Therefore, it can act on the level of fibrils, rather than on the whole shock structure, and also within the frequency window of the expected oscillations. In other words, this type of perturbation has the appropriate amplitude, spatial and temporal scales to potentially disrupt the synchronization and hence affect the global quasi-periodic signature.

\subsubsection{Shape of the impacting surface}

Finally, even in the case of a homogeneous accretion stream and a static chromosphere, the starting time for each fibril oscillation is determined by the shape of the surface of first impact. For instance, if the center of the funneled gas cross-section hits the star earlier that its outer parts by an interval comparable to, or larger than, $\tau_{\text {cool }}$ then the formation and collapse of the fibrils will be out of phase.

\section{Numerical study}

The previous rough estimates indicate that introducing perturbations in the accretion shock system can potentially suppress the predicted periodic emission. In this section, we explore the actual effects of several types of realistic perturbations by performing numerical simulations and studying the time-dependent shock structure. We illustrate the involved processes in the dynamics, showing also the role of the magnetic field.

\subsection{Random density perturbations in the stream}

Figure 6 shows a snapshot of the spatial distribution of the physical quantities for the high plasma- $\beta$ model Rnd20. Three regions can be identified from top to bottom: the tenuous and cold accretion stream, the denser post-shock region of a few million Kelvin, and the relatively colder isothermal chromosphere 

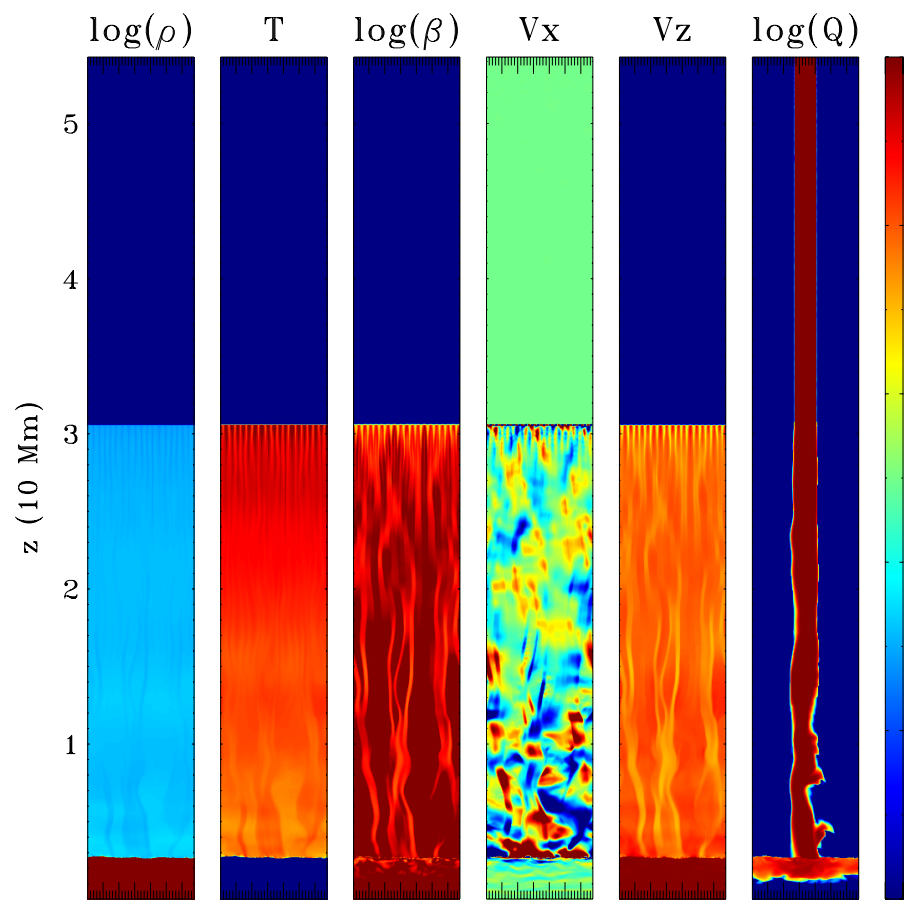

Fig. 6. Density, temperature, plasma- $\beta$, and velocity distributions at $t=10 \mathrm{ks}$ for the weak magnetic field model Rnd20. The colors blue/red are used for low/high values, and in particular $\rho \in\left[10^{-13}, 10^{-11}\right] \mathrm{g} \mathrm{cm}^{-3}$, $T \in[0,4] \mathrm{MK}, \beta \in\left[10^{-2}, 10^{2}\right], v_{x} \in[-5,5] \mathrm{km} \mathrm{s}^{-1}, v_{z} \in$ $[-500,0] \mathrm{km} \mathrm{s}^{-1}$, and $Q \in[0,1]$. The $1 \%$ density perturbations that are applied to this model are too weak to be visible. Due to the saturation of the density color, the exponential profile of the chromosphere cannot be seen.

which is described by an exponential density profile. As expected, the post-shock region resembles closely the unperturbed case (NoPert) because the magnetic field is not strong enough to prevent pressure fluctuations from being smoothed out. This produces a chaotic motion of a few $\mathrm{km} \mathrm{s}^{-1}$ as it can be seen in the panel of $v_{x}$ whose extrema correspond to $\pm 0.01 v_{\text {acc }}$. The vertical velocity component of the shocked material has a similar signature but this is not apparent in the corresponding plot due to the different color range used $\left( \pm v_{\text {acc }}\right)$. Furthermore, the accreted material is reposited all over the surface of the chromosphere, as indicated by the tracer, $Q$.

Figure 7 displays the same physical variables as Fig. 6 but for the low plasma- $\beta$ model Rnd500. Notice the fibril structure of the shocked material which is the result of the magnetic confinement of the plasma inside vertical flux tubes. The temporal evolution of each fibril is independent of its neighbours; some of them are forming, e.g. regions on the right part of each panel, whereas others are collapsing, e.g. regions on the left. This can be seen by looking at the vertical distribution of the temperature of a given fibril. A forming fibril has a rather uniform temperature along $z$. On the contrary, a collapsing fibril may have $T$ varying by orders of magnitude along its height. The horizontal speed component is zero almost everywhere, showing that plasma mixing cannot take place easily in the low plasma- $\beta$ regime. For the same reason, the tracer shows that the material accretes strictly vertically and any further mixing can take place only deeper in the chromosphere where the thermal pressure is stronger than the magnetic one. Finally, note that despite the small perturbation amplitude, a phase shift does develop among fibrils.

Figure 8 illustrates the dependence of the fibril size on the strength of the magnetic field. Although quantitative analysis is
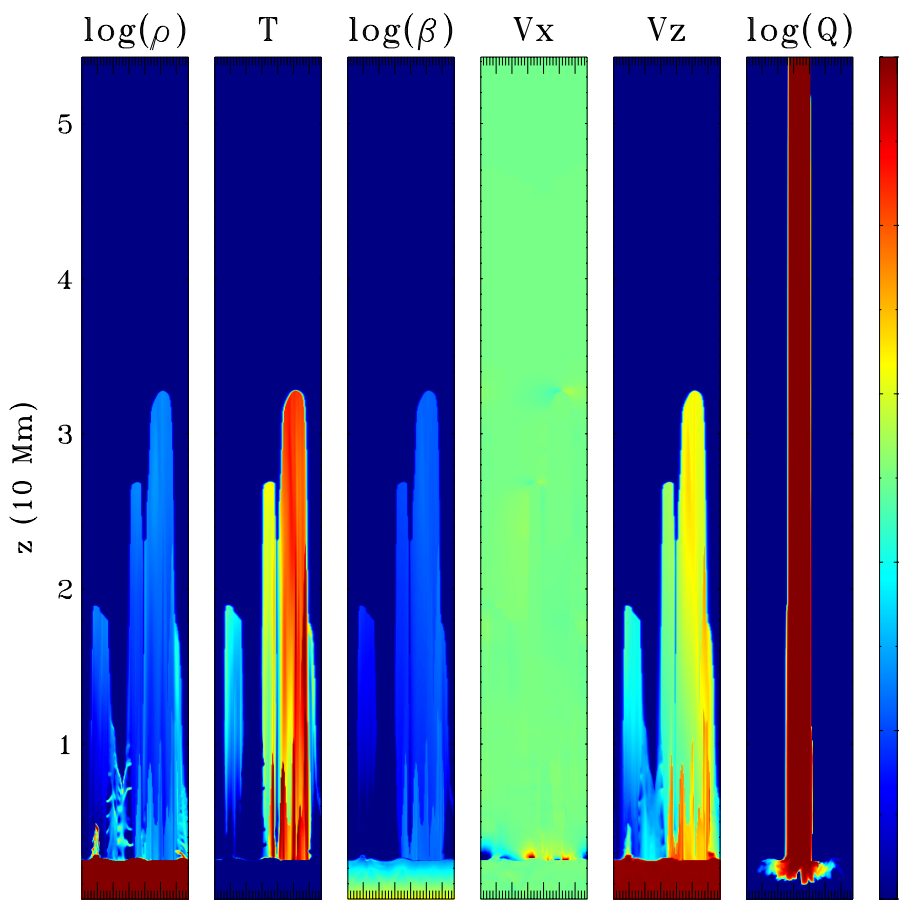

Fig. 7. Density, temperature, plasma- $\beta$, velocities, and tracer distibutions at $t=10 \mathrm{ks}$ for the strong magnetic field case Rnd500. The range of each plotted quantity is: $\rho \in\left[10^{-13}, 10^{-11}\right] \mathrm{g} \mathrm{cm}^{-3}, T \in[0,2] \mathrm{MK}$, $\beta \in\left[10^{-2}, 10^{2}\right], v_{x} \in[-5,5] \mathrm{km} \mathrm{s}^{-1}, v_{z} \in[-500,0] \mathrm{km} \mathrm{s}^{-1}$, and $Q \in[0,1]$.

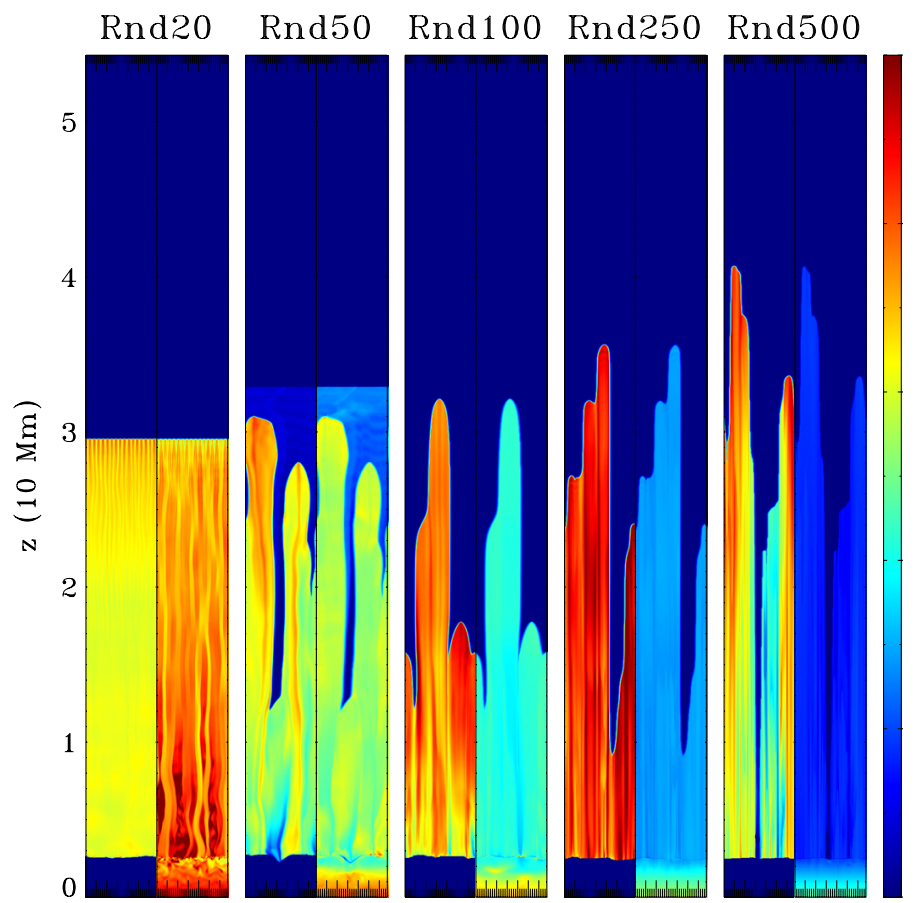

Fig. 8. Distributions of the temperature (on the left of each panel) and the plasma- $\beta$ (on the right of each panel) for the models NoPert, Rnd20, Rnd50, Rnd100, Rnd250, and Rnd500 (left to right). The plotted ranges are: $T \in[0,5] \mathrm{MK}$ and $\beta \in\left[10^{-3}, 10^{3}\right]$. The snapshots do not correspond to the same time, but each one was chosen such that the fibril structure becomes more apparent. Note also that the aspect ratio is not the same as the rest of the figures: these plots are compressed vertically by a factor of 1.5 .

required to verify the approximate relation of Eq. (20), namely $d \propto 1 / B_{z}$, it seems to be valid to zeroth order (see Sect. 3.3.2). 
T. Matsakos et al.: Suppressing the periodic emission of YSO accretion shocks

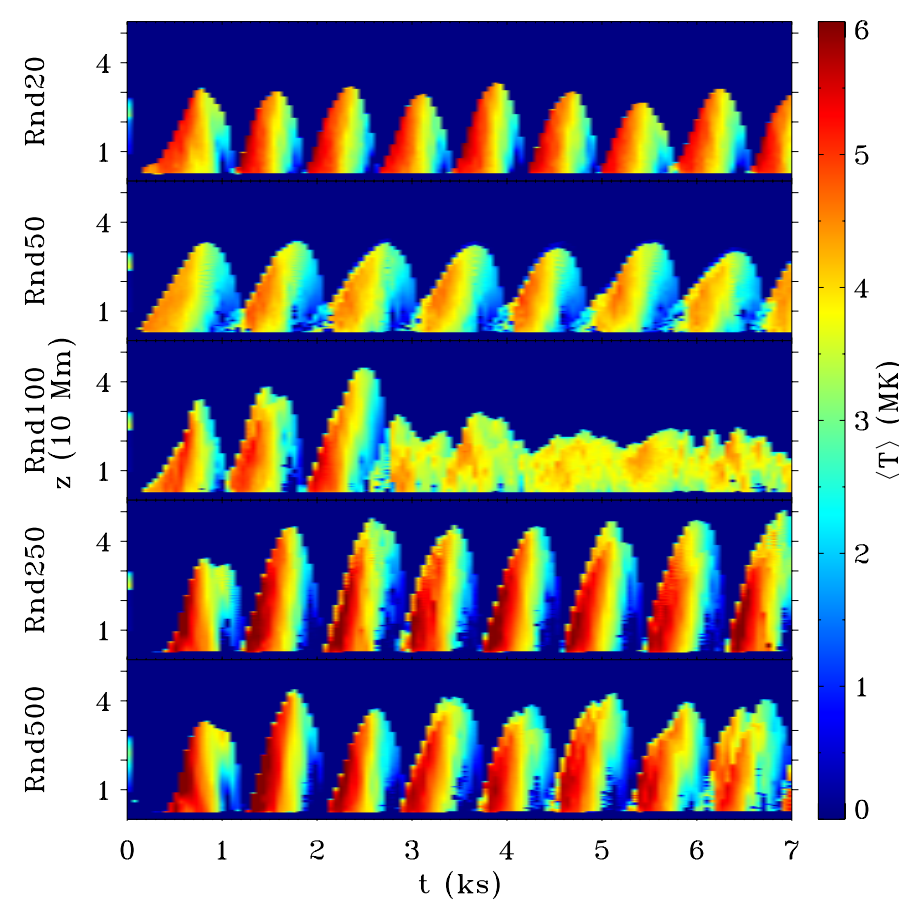

Fig. 9. Emission-measure weighted temperature versus $z$ and $t$ for the models that consider $1 \%$ random density perturbations in the stream, i.e. Rnd20, Rnd50, Rnd100, Rnd250, and Rnd500 (top to bottom).

Note that the size of the fibrils does not depend on the perturbation length scale, which for these models is the cell width $\Delta x$, but only on the plasma- $\beta$ value. This is also confirmed by all other simulations of Table 1 . In addition, the fibrils seem to have a finer structure at the base close to the chromosphere and a thicker and rounded shape at their head.

Furthermore, by assuming an accretion stream radius of $5 \times 10^{4} \mathrm{~km}$ (Orlando et al. 2010), the computational box considered here represents approximately one hundredth of its diameter. This provides an estimate on the total number of fibrils that can form and also shows the overall complexity of the postshock region even in the case of uniform magnetic fields. On the other hand, we have verified that our simulations capture correctly the width of the fibrils since it remains the same when increasing the resolution. Nevertheless, lower plasma- $\beta$ values would demand smaller cell sizes due to the anticipated thinner fibrils. The above two points imply that simulating the entire stream together with the highly structured post-shock region is a rather computationally expensive task.

In order to quantify the effects that the $1 \%$ random density fluctuations have on the periodicity of the system, we plot in Fig. 9 the emission-measure weighted temperature, $\langle T(z, t)\rangle$, for this class of models. Overall, and despite the formation of fibrils in the low plasma- $\beta$ cases, a strong periodic signature can be observed in the cases Rnd20, Rnd50, Rnd250, and Rnd500. This is because the applied perturbation is small and it also has a zero net effect along $x$. In other words, fluctuations at each horizontal position cancel in time, making each flux tube, on average, identical to the rest. Recall that bringing the fibrils out of phase is not an unstable process and therefore their oscillations remain generally synchronized with only a small phase difference attributed to the small density fluctuations. Here, a stronger and less symmetric perturbation is required to produce an adequate phase shift among adjacent fibrils.
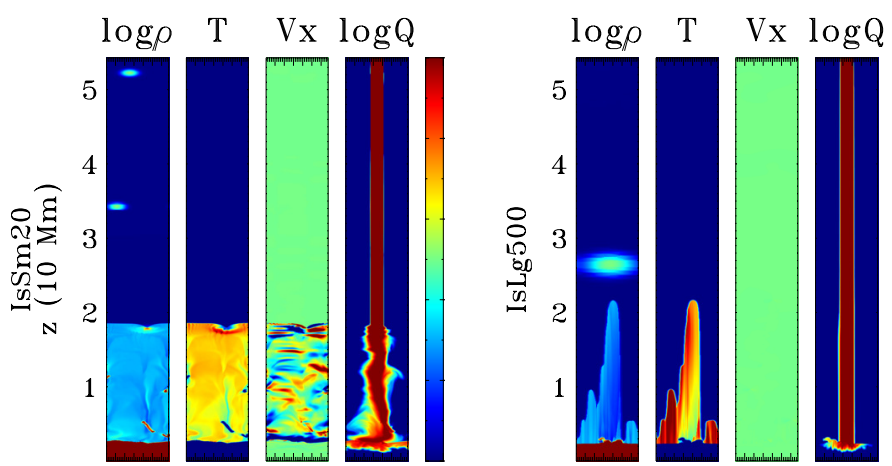

Fig. 10. Density, temperature, horizontal velocity, and the fluid tracer for the isolated clump models IsSm20 (left panels) and IsLg500 (right panels). Blue/red color corresponds to low/high values, i.e. $\rho \in$ $\left[10^{-13}, 10^{-11}\right] \mathrm{g} \mathrm{cm}^{-3}, T \in[0,5] \mathrm{MK}, v_{x} \in[-50,50] \mathrm{km} \mathrm{s}^{-1}$, and $Q \in$ $[0,1]$. The clumps, visible as elliptical spots in density distributions, are circular but appear elongated due to the compression of the vertical direction for visualization purposes.

Nevertheless, model Rnd100 seems to lose its periodic signature in the long term. Its plasma- $\beta$ at the post-shock region is $\beta \sim 1$ which suggests that the dynamics are neither dominated by purely hydro processes nor by the magnetic field alone, as it is the case for the other models. Instead, there is a complex interplay between the two that gives rise to $2 \mathrm{D}$ mechanisms which in turn disrupt the global periodic behavior even for such relatively small perturbations. Finally, by comparing Figs. 5 and 9 we observe that the maximum height attained by the perturbed cases is lower than that of the unperturbed, and also that their oscillation period is slightly shorter. For the high plasma- $\beta$ cases this is due to the inhomogeneities produced in the hot slab, where the locally denser regions are susceptible to stronger energy losses. Effectively, this reduces the average $\tau_{\text {cool }}$ as it is also observed and discussed in Sutherland et al. (2003). On the other hand, even though the low plasma- $\beta$ cases do reach a higher height than the weak $\boldsymbol{B}$ models, the small deformation of the flux tubes by the confined hot plasma invalidates the $x$-symmetry and, as a result, the fibrils do not attain the height of NoPert.

\subsection{Isolated clumps or fully clumped stream}

Figure 10 displays the physical quantities of two models that incorporate isolated clumps into the stream, Is Sm 20 and IsLg500. On the left, two small clumps are visible in the infalling gas and a third one that has just hit the reverse shock. The postshock region is hotter where the clump interacts with the reverse shock. There, the basic physics is similar to that for the interaction of a supernova remnant shock with a cloud of the ISM, where the major factor is the density contrast of the clump with respect to the surrounding medium (e.g. Klein et al. 1994; see also Orlando et al. 2008, for a MHD study including the effects of anisotropic thermal conduction). The shock-clump interaction generates transmitted (into the clump) and reflected (into the post-shock material of the slab) shocks, whose temperature depends on the density contrast of the clump. The darkest portion of the shock front in the second panel of Fig. 10 is the shocked clump material and the red structure that surrounds it is the bow shock. The latter propagates into the shocked stream with a higher temperature than that of its environment. A strong velocity field appears locally, with the value of $v_{x}$ reaching almost half of the accretion speed, $v_{\text {acc }}$. In fact, the hot slab shows overall much higher velocity values of chaotic motion than the 


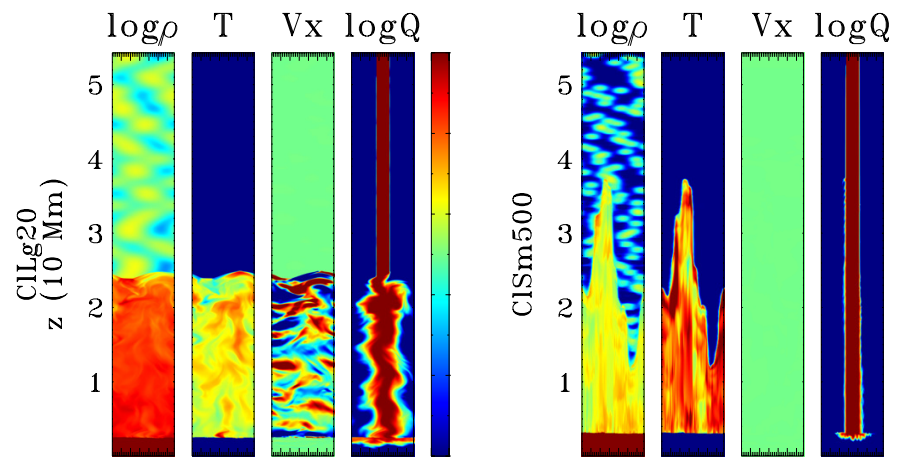

Fig. 11. Density, temperature, horizontal velocity, and the fluid tracer for models ClLg20 (left panels) and $\mathrm{ClSm} 500$ (right panels), which assume a fully clumped accretion stream. The plotted value ranges are: $\rho \in\left[10^{-14}, 10^{-12}\right] \mathrm{g} \mathrm{cm}^{-3}, T \in[0,5] \mathrm{MK}, v_{x} \in[-50,50] \mathrm{km} \mathrm{s}^{-1}$, and $Q \in[0,1]$.

model Rnd20; here the velocity is plotted in the range $\pm 0.1 v_{\text {acc }}$ with larger values saturating to dark blue or dark red, respectively. On the other hand, the strong magnetic field of model IsLg500 (on the right) does not allow pressure homogenization and hence there is no plasma mixing. The formation of fibrils is again evident in the system, but this time their oscillations are effectively brought out of phase. This is due to the large density contrast of the clumps which significantly modify the local value of $\tau_{\text {cool }}$

The general features observed in the isolated clump models are also seen in the cases of fully clumped streams. Figure 11 displays the spatial distribution of the physical quantities for ClLg20 and C1Sm500. In these cases too, the dynamics of the post-shock region depends on the strength of the magnetic field and not on the type of perturbation. Even though the average density of the infalling gas, and hence the cooling time, is different than before, the temporal evolution is generally similar; the high plasma- $\beta$ models show chaotic motion and mixing whereas the low plasma- $\beta$ cases a fibril structure.

The periodic signature of all the models that consider clumps can be derived from Fig. 12. The presence of dense clumps increases the local cooling efficiency of the post-shock region and as a result the emission may vary strongly with $z$. The difference in the frequencies (when there is one) and the maximum height attained are attributed to the value of the average density of the accreting material. In particular, the high plasma- $\beta$ models IsSm2 $\theta, C 1 S m 2 \theta$, and ClLg2 $\theta$ maintain a quasi-periodic oscillatory character. On the other hand, the strong magnetic field models have their fibrils oscillating fully out of phase. As a result, the periodic behavior is heavily disrupted in most cases and suppressed in the model IsLg500. We note that the limited width and dimensionality of the box is contributing to these noisy profiles. If we had explored the whole accretion stream cross section or performed 3D simulations, the sum of individual out-ofphase fibril contributions would have provided a much smoother profile.

\subsection{Perturbations in the chromosphere}

Models ChrFlx\# show the same magnetic-field-dependent features of the accretion shock structure that were discussed earlier, despite the intrinsically different perturbation type. The sinusoidal spatial profile of the chromospheric pressure fluctuations generates local vertical velocities in all three cases and also a horizontal motion when the magnetic field is weak enough. As
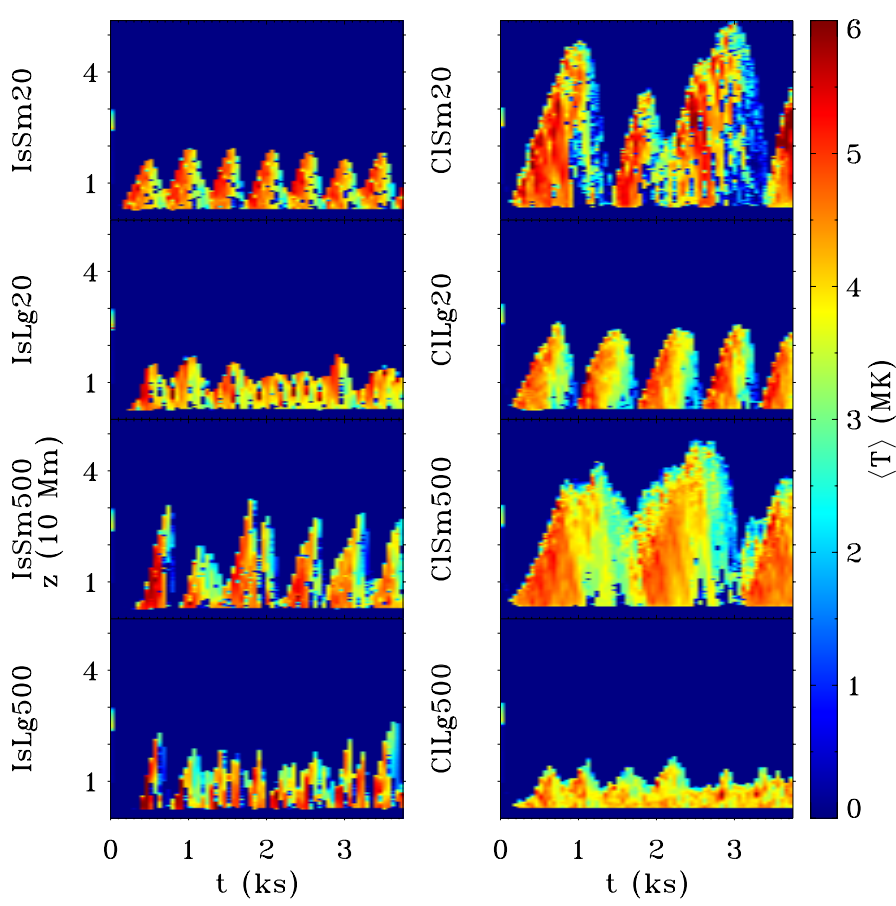

Fig. 12. Emission-measure weighted temperature for the models that assume isolated clumps (left panels) and the a fully clumped stream (right panels).
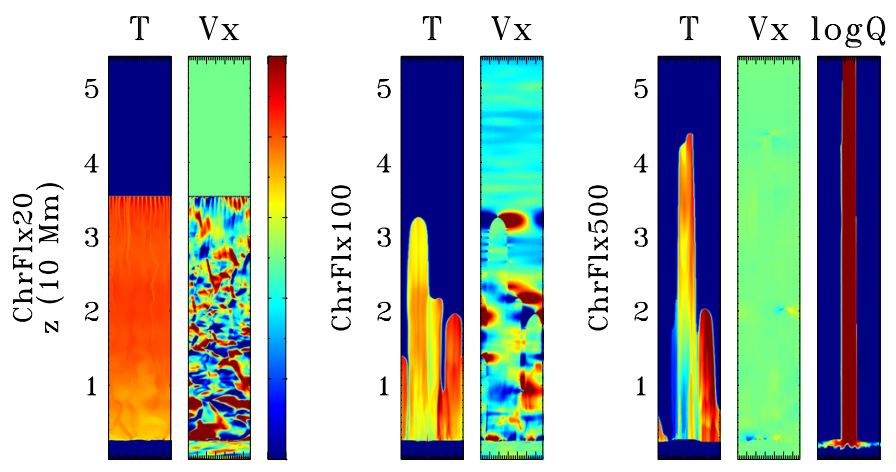

Fig. 13. Spatial distribution of the temperature and of the horizontal velocity component for the models that consider an energy flux in the chromosphere. Plotted ranges: $T \in[0,5] \mathrm{MK}, v_{x} \in[-5,5] \mathrm{km} \mathrm{s}^{-1}$, and $Q \in[0,1]$.

it can be seen in Fig. 13, the upwards travelling sonic waves disrupt the lower edge of the post-shock region leading to a similar $\boldsymbol{B}$-dependent morphology of the shocked material as the rest of the models in Table 1 . The $v_{x}$ component is plotted in the range $\pm 0.01 v_{\text {acc }}$ but we note that the values of the chaotic motion can be by an order of magnitude larger close to the chromosphere. Furthermore, notice that $\mathrm{ChrF} 1 \times 100$ displays a non-zero value of $v_{x}$ in the stream. This effect is created from MHD waves that travel along the accretion flow and force the material to follow the wavy structure of the magnetic field lines. Therefore, in the low plasma- $\beta$ regime, any type of chromospheric fluctuations can also perturb the stream apart from the accretion shock. This effect is present in all models, even though weaker.

The top three panels of Fig. 14 show the emission-measure weighted temperature versus time for the three models considering perturbations of chromospheric origin. Once again we confirm that the periodicity is present when the magnetic field is weak. In the same context, the independent fibril oscillations of the low plasma- $\beta$ case hide any well-defined temporal patterns. 


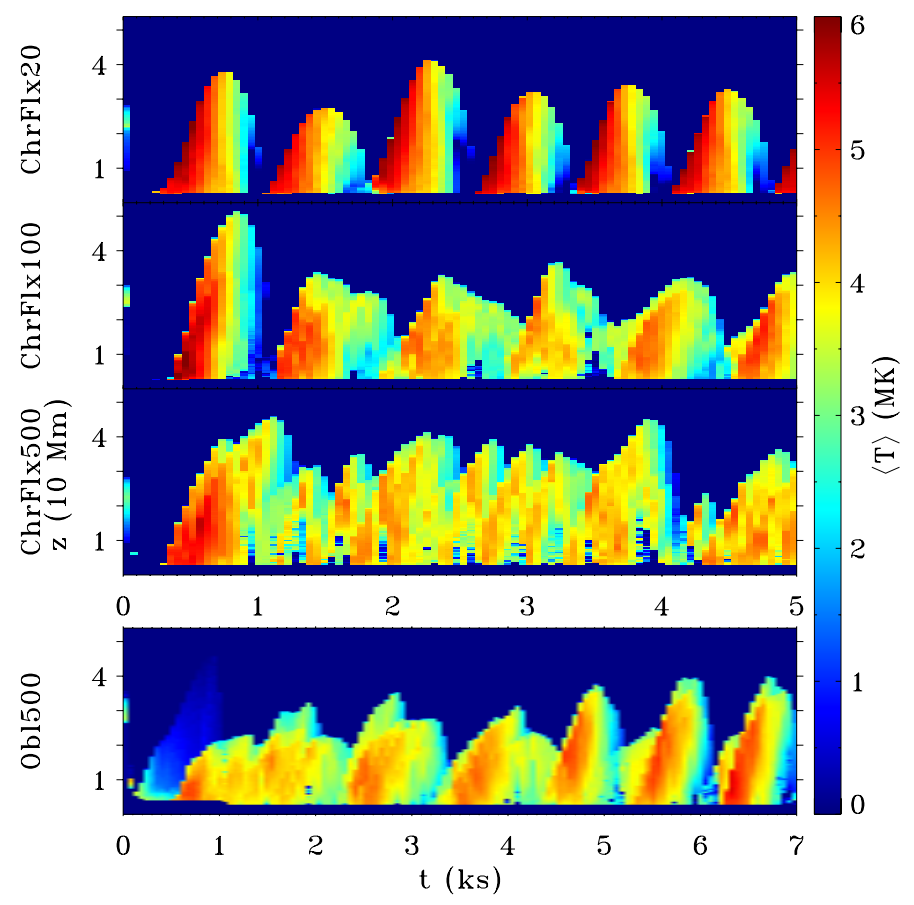

Fig. 14. Emission-measure weighted temperature for the models that consider fluctuations in the chromosphere (top three) and for the case of an oblique surface of impact (bottom).

\subsection{Oblique impacting surface}

Finally, the bottom panel of Fig. 14 presents model Obl500. By definition, this case is prepared such that among flux tubes there is a phase shift on the starting time of the fibril oscillations. However, once the whole stream has entered the box, no further perturbations are introduced. The plot suggests that although the fibrils are initially out of phase, they gradually get synchronized. This implies that a coordinated oscilation of the fibrils is a stable state and that the weak interaction between the flux tubes favors the synchronization of the system.

\section{Summary - conclusions}

In the literature, theoretical studies and 1D numerical simulations predict an oscillatory behavior in the emission of accretion shocks. However, observational data appear not to confirm such an expectation. In this paper, we have explored whether the presence of perturbations could disrupt or even suppress this periodic signature. We have performed 2D MHD simulations of the interior of the accretion flow to investigate the effects of several realistic perturbations, such as a clumped stream or fluctuations in the chromosphere. We have explored a wide range of magnetic field values in order to understand its role in the dynamics. Our conclusions are summarized as follows:

- The structure of the post-shock region is found to depend strongly only on the plasma- $\beta$ value and only very weakly on the nature of the perturbation. In particular, a relatively strong magnetic field confines the plasma in flux tubes and leads to the formation of fibrils. Their size depends on the value of $\boldsymbol{B}$ and their evolution follows roughly a quasiperiodic oscillation that is almost independent of their neighbours. In the high plasma- $\beta$ regime, the post-shock region shows chaotic motion and plasma mixing with the value of local velocity determined by the applied perturbation.
- In the cases where the magnetic field is weak, the emission maintains a strong periodic signature. This is because the pressure fluctuations that appear in the post-shock region, due to the applied perturbations, are smoothed out and the entire hot slab forms and recollapses as a whole. However, both the oscillation frequency and the height reached by the reverse shock are lower than those of the unperturbed case due to the more efficient cooling, a byproduct of chaotic motion and the inhomogeneities it produces.

- For strong magnetic fields, the global periodicy in the emission can be suppressed if the fibrils oscillate out of phase. This requires a perturbation that can effectively modify the cooling time and/or the trigger time among adjacent flux tubes. We point out that since observations of protostars suggest high magnetic field values, the low plasma- $\beta$ models we have investigated in this paper are expected to be the most relevant for real YSOs.

- In general, for a perturbation to have an effect on the system, it has to have a time scale comparable to the cooling time, a length scale comparable to the fibril width, and an adequate amplitude. All three criteria seem to be fulfilled by the typical perturbations expected to be present in the accretion shock system.

In order to compare these theoretical accretion models with observations, adequate radiative transfer post-processing treatment is required to determine spectral signatures. As part of further investigations, we will make use of the 3D IRIS code (Ibgui et al. 2013), to predict the temporal, spatial, angular, and spectral distributions of the radiation that emerges from such 2D/3D accretion shock simulations. It will be also interesting to address the possibility that the accretion flows are fragmented, as recently suggested from solar observations (Reale et al. 2013).

Acknowledgements. We would like to thank an anonymous referee for the helpful comments on the manuscript. The authors are grateful to A. Ciardi for his help and suggestions during the preparation of this study. We would also like to thank F. Thais for his support as well as A. Mignone, M. Flock, S. Matt, G. Herczeg, J. Bouvier, S. Cabrit, A. Maggio, A. Bonanos, T. Katsiyannis, R. Keppens, C. Xia, and R. Pinto for fruitful discussions. This work was supported by the ANR STARSHOCK project (ANR-08-BLAN-0263-07-2009/2013) and was granted access to the HPC resources of CINES under the allocation 2012c2012046943 made by GENCI (Grand Equipement National de Calcul Intensif).

\section{References}

Argiroffi, C., Maggio, A., \& Peres, G. 2007, A\&A, 465, L5

Bary, J. S., Matt, S. P., Skrutskie, M. F., et al. 2008, ApJ, 687, 376

Beck, C., Khomenko, E., Rezaei, R., \& Collados, M. 2009, A\&A, 507, 453

Beck, C., Rezaei, R., \& Puschmann, K. G. 2013, A\&A, 553, A73

Bello González, N., Flores Soriano, M., Kneer, F., \& Okunev, O. 2009, A\&A, 508, 941

Bohn, H. U. 1984, A\&A, 136, 338

Bouvier, J., Alencar, S. H. P., Boutelier, T., et al. 2007a, A\&A, 463, 1017

Bouvier, J., Alencar, S. H. P., Harries, T. J., Johns-Krull, C. M., \& Romanova, M. M. 2007b, Protostars and Planets V, 479

Brickhouse, N. S., Cranmer, S. R., Dupree, A. K., Luna, G. J. M., \& Wolk, S. 2010, ApJ, 710, 1835

Calvet, N., \& Gullbring, E. 1998, ApJ, 509, 802

Chevalier, R. A., \& Imamura, J. N. 1982, ApJ, 261, 543

Cowie, L. L., \& McKee, C. F. 1977, ApJ, 211, 135

Donati, J.-F., Jardine, M. M., Gregory, S. G., et al. 2007, MNRAS, 380, 1297

Drake, J. J., Testa, P., \& Hartmann, L. 2005, ApJ, 627, L149

Drake, J. J., Ratzlaff, P. W., Laming, J. M., \& Raymond, J. 2009, ApJ, 703, 1224

Dupree, A. K., Brickhouse, N. S., Cranmer, S. R., et al. 2012, ApJ, 750, 73

Gullbring, E. 1994, A\&A, 287, 131

Gullbring, E., Barwig, H., Chen, P. S., Gahm, G. F., \& Bao, M. X. 1996, A\&A, 307,791

Günther, H. M., Liefke, C., Schmitt, J. H. M. M., Robrade, J., \& Ness, J.-U. 2006, A\&A, 459, L29 
Günther, H. M., Lewandowska, N., Hundertmark, M. P. G., et al. 2010, A\&A, 518, A54

Hartmann, L., Hewett, R., \& Calvet, N. 1994, ApJ, 426, 669

Ibgui, L., Hubeny, I., Lanz, T., \& Stehlé, C. 2013, A\&A, 549, A126

Johns-Krull, C. M. 2007, ApJ, 664, 975

Judge, P. 2006, in Solar MHD Theory and Observations: A High Spatial Resolution Perspective, eds. J. Leibacher, R. F. Stein, \& H. Uitenbroek, ASP Conf. Ser., 354, 259

Kashyap, V., \& Drake, J. J. 2000, Bull. Astron. Soc. India, 28, 475

Kastner, J. H., Huenemoerder, D. P., Schulz, N. S., Canizares, C. R., \& Weintraub, D. A. 2002, ApJ, 567, 434

Klein, R. I., McKee, C. F., \& Colella, P. 1994, ApJ, 420, 213

Koenigl, A. 1991, ApJ, 370, L39

Koldoba, A. V., Ustyugova, G. V., Romanova, M. M., \& Lovelace, R. V. E. 2008, MNRAS, 388, 357

Lamzin, S. A. 1998, Astron. Rep., 42, 322

Lamzin, S. A. 1999, Astron. Lett., 25, 430

Langer, S. H., Chanmugam, G., \& Shaviv, G. 1981, ApJ, 245, L23

Mignone, A. 2005, ApJ, 626, 373

Mignone, A., Bodo, G., Massaglia, S., et al. 2007, ApJS, 170, 228

Mignone, A., Zanni, C., Tzeferacos, P., et al. 2012, ApJS, 198, 7

Orlando, S., Bocchino, F., Reale, F., Peres, G., \& Pagano, P. 2008, ApJ, 678, 274

Orlando, S., Sacco, G. G., Argiroffi, C., et al. 2010, A\&A, 510, A71
Orlando, S., Bonito, R. Argiroffi, C., et al. 2013, A\&A, submitted Ramachandran, B., \& Smith, M. D. 2005a, MNRAS, 362, 1353 Ramachandran, B., \& Smith, M. D. 2005b, MNRAS, 357, 707

Reale, F., Orlando, S., Testa, P., et al. 2013, Science, 341, 251

Robrade, J., \& Schmitt, J. H. M. M. 2007, A\&A, 473, 229

Romanova, M. M., Ustyugova, G. V., Koldoba, A. V., Wick, J. V., \& Lovelace, R. V. E. 2003, ApJ, 595, 1009

Romanova, M. M., Ustyugova, G. V., Koldoba, A. V., \& Lovelace, R. V. E. 2004 ApJ, 610, 920

Romanova, M. M., Kulkarni, A. K., \& Lovelace, R. V. E. 2008, ApJ, 673, L171

Sacco, G. G., Argiroffi, C., Orlando, S., et al. 2008, A\&A, 491, L17

Sacco, G. G., Orlando, S., Argiroffi, C., et al. 2010, A\&A, 522, A55

Safier, P. N. 1998, ApJ, 494, 336

Schmitt, J. H. M. M., Robrade, J., Ness, J.-U., Favata, F., \& Stelzer, B. 2005, A\&A, 432, L35

Smith, R. K., Brickhouse, N. S., Liedahl, D. A., \& Raymond, J. C. 2001, ApJ, 556, L91

Spitzer, L. 1962, Physics of Fully Ionized Gases (New York: Interscience)

Stelzer, B., \& Schmitt, J. H. M. M. 2004, A\&A, 418, 687

Sutherland, R. S., Bicknell, G. V., \& Dopita, M. A. 2003, ApJ, 591, 238

Telleschi, A., Güdel, M., Briggs, K. R., Audard, M., \& Scelsi, L. 2007, A\&A, 468,443

Toth, G., \& Draine, B. T. 1993, ApJ, 413, 176

Ulrich, R. K. 1976, ApJ, 210, 377 